\title{
SPECTRAL ANALYSIS OF BREAKDOWN AT OR NEAR RF WINDOWS
}

\author{
D. L. Borovina", J. M. Gahl, UNM, Albuquerque, NM \\ D. Rees, LANL, Los Alamos, NM
}

\begin{abstract}
The University of New Mexico (UNM) klystron test stand can generate microwave pulses up to 2-ms long with a frequency of $850 \mathrm{MHz}$ and a peak power of $1.26 \mathrm{MW}$. For these experiments, the klystron output was used to simulate field breakdown near or on the surface of RF vacuum windows. RF-driven discharges were initiated in vacuum waveguide sections between copper electrodes. Data were gathered for discharges in the gap between the electrodes and for discharges between the electrodes and across the surface of the ceramic alumina. The generated optical signals were captured through three different windows (sapphire, crystal quartz, and $\mathrm{NaCl}$ ) using different photo-multiplying tubes (PMTs). Issues addressed include a partial analysis of the spectra from metal/metal and metal/ceramic arcs and an investigation of arc precursor emission. The results of these preliminary tests will be used to optimize selection of optical components in arc detection systems.
\end{abstract}

\section{INTRODUCTION}

$\mathrm{RF}$ window failure in particle accelerators is an issue of great concern. Arc detection systems utilizing photomultiplying tubes (PMTs) are commonly used to observe and possibly avoid this process. This work seeks to characterize the response of PMTs to RF-driven metal/metal and metal/ceramic arcs through a variety of arc detector window materials. We intend to characterize these arc detection systems with regard to their intensity, duration, and promptness of response.

\section{EXPERIMENTAL LAYOUT}

\subsection{Microwave Generation}

Extensive work was conducted on the construction of a high power, Klystron-based, microwave test stand at the University of New Mexico. This test stand was built to investigate RF windows, waveguide components, and new diagnostics that will predict or detect quickly window or waveguide breakdown. The test stand was used to characterize RF-load film resistors for the Low Energy Demonstration Accelerator (LEDA) at Los Alamos with the results reported separately at this meeting [1].

"Work supported by the U. S. Department of Energy under Contract No. DE-FG04-97AL77993.

"Email: dlborovina@aol.com
The test facility depends heavily on equipment transferred to the University of New Mexico from Los Alamos National Laboratory (LANL) and DOE and is housed in a 2500 square foot high-bay facility on UNM's south campus.

A water cooling system as well as an oil storage and transfer system has been installed in the high bay. A screen room has been constructed to house diagnostic equipment. An Allen Bradley rack controls a modulator which is driven by a Universal Voltronix power supply. This arrangement, shown in Figure 1, was designed for a high-power, 850-MHz TH2138 Klystron tube, which drives the test stand with pulses up to 2-ms long and a peak power of $1.26 \mathrm{MW}$.

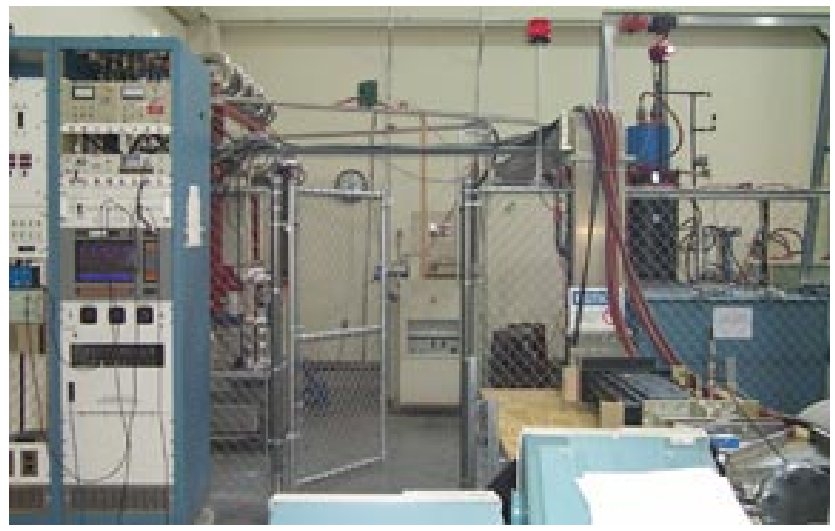

Figure 1: The University of New Mexico klystron test stand.

\subsection{Microwave Breakdown Experiments}

For these experiments, the test stand's output was used to simulate field breakdown near or on the surface of RF vacuum windows. RF driven discharges were initiated in vacuum waveguide sections between copper electrodes. Breakdown was achieved with a RF drive power of two to three hundred kilowatts. An isolator was utilized to protect the klystron from the RF reflected by the arcs generated.

Data were gathered for discharges struck in the vacuum gap between the electrodes (metal/metal arcs) and for discharges between the electrodes and across the surface of the ceramic alumina (metal/ceramic arcs). The generated optical signals were captured through three different detector window materials (sapphire, crystal quartz, and $\mathrm{NaCl}$ ) using photo-multiplying tubes that had different spectral responses. 
Two Hamamatsu 38-mm-diameter PMTs were used [2]. The 6199 type is a general purpose PMT that has a spectral response of $300-650 \mathrm{~nm}$, covering most of the visible range and the near ultra violet. The typical rise time of the tube is $2.8 \mathrm{~ns}$ with an electron transit time of $40 \mathrm{~ns}$. The second photo-multiplying tube used was the 7102 type with a spectral response of $400-1200 \mathrm{~nm}$, covering the visible range and the near infrared. The typical rise time of the tube is $2.2 \mathrm{~ns}$ with average electron transit time of $37 \mathrm{~ns}$.

The three different window materials have a flat spectral transmission characteristic over the spectral response of both tubes. The different window materials were used to see their potential effect on the magnitude of light transmission to both of the PMTs. The quartz window was approximately $3.3-\mathrm{mm}$ thick, the sapphire window 1.1-mm thick, and the $\mathrm{NaCl}$ window was approximately $4.3-\mathrm{mm}$ thick.

Data were taken under all possible combinations. Both PMTs viewed metal/metal and metal/ceramic discharges through all three window types. Of course experimental conditions such as gap separation and electrode configuration were kept as constant as possible. RF power levels of $200-300 \mathrm{~kW}$ were required to drive RF breakdown between the electrodes. At these power levels, electrodes were regularly degraded and required adjustment. Due in part to the concern that large amounts of RF energy were being reflected back at the protection isolator and the klystron, data acquisition was limited for this preliminary study to roughly 100 discharge events.

\section{EXPERIMENTAL RESULTS}

No significant variation in magnitude of signal was observed though the various window types. Nor did the two different PMTs show a variation in duration of light emitted. The most interesting effect we observed was a difference in the promptness of the response of the two PMTs.

When viewing metal/ceramic arcs, the 7102 type PMT (with a spectral response into the infrared) detected light on average approximately $18 \mu$ s earlier than the 6199 type PMT. Figure 2 shows the reflected RF power from a metal/ceramic arc with the corresponding response of the 7102 PMT. Figure 3 shows the reflected RF power from a $\mathrm{metal} / \mathrm{ceramic}$ arc with the corresponding response of the 6199 PMT.

The effect was not as significant for metal/metal arcs but still seemed to exist. When viewing metal/metal arcs, the 7102 type PMT detected light on average approximately $6 \mu \mathrm{s}$ earlier than the 6199 type photomultiplying tube.

As described earlier from the manufacture's data sheets, electrical differences in the two tubes should only account for response variations on the order of a few nanoseconds.

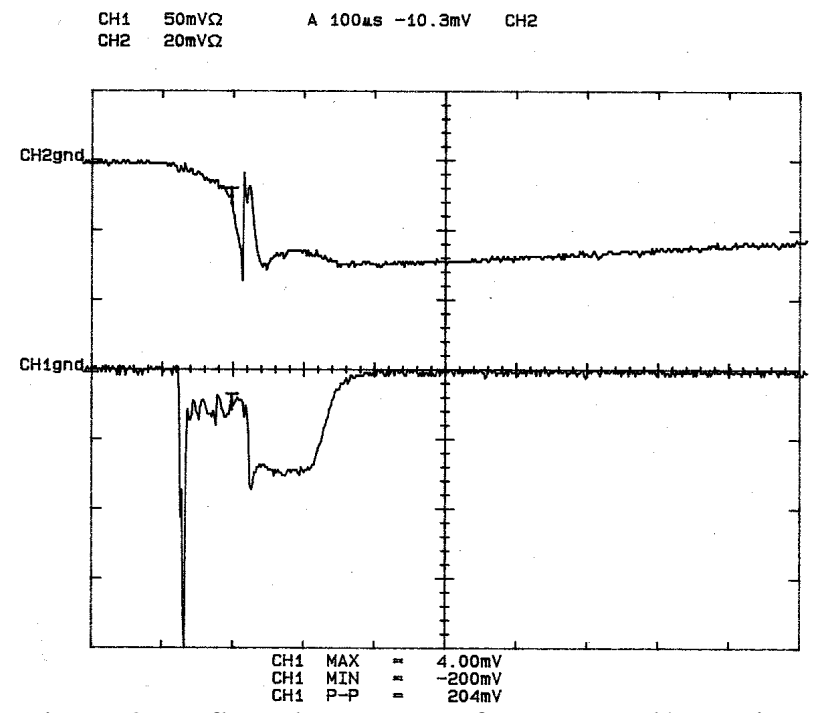

Figure 2: Reflected RF power from a metal/ceramic arc with the corresponding response of the 7102 PMT (100 $\mu \mathrm{s} / \mathrm{div})$.

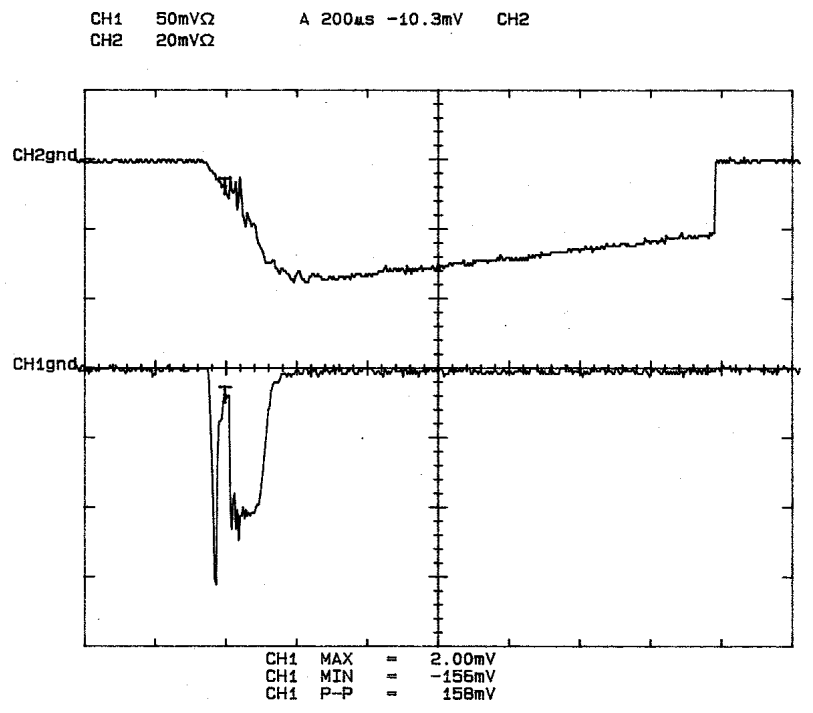

Figure 3: Reflected RF power from a metal/ceramic arc with the corresponding response of the 6199 PMT (200 $\mu$ s/div).

\section{CONCLUSIONS}

A variety of conclusions and recommendations for future investigations can be drawn from the results of this preliminary study, namely:

1. Infrared radiation may be emitted from $\mathrm{RF}$ window arcs, particularly metal/ceramic arcs, earlier than visible radiation.

2. To insure the most prompt response to a window arc, detection systems should be able to detect infrared radiation. 
3. A more comprehensive spectroscopic study of metal/metal and metal/ceramic arcs may lead to the ability of arc detection systems to delineate such breakdowns.

\section{ACKNOWLEDGEMENTS}

We acknowledge and thank Lee Terry of UNM for his help in experimental preparations and high-voltage safety precautions.

\section{REFERENCES}

[1] D. L. Borovina, J. M. Gahl, S. Humphries, Jr., D. Rees, L. Toole, "Experimental and Analytic Studies of an RF Load Resistor", presented at the Particle Accelerator Conference PAC'99, New York, April 1999. [2] Photomultiplier Tubes, Catalog No. TPMO 0002E04, Hamamatsu Corporation, Bridgewater, N.J., August 1995. 